\title{
Manejo de fracturas inestables de pelvis con clamp en $\mathrm{C}$
}

\author{
C clamp for unstable pelvic \\ fracture management
}

\author{
Luis Justino Fernández Palomo, ${ }^{\text {Ramón González Pola* }}$
}

Citar como: Fernández PLJ, González PR. Manejo de fracturas inestables de pelvis con clamp en C. An Med ABC. 2021 ; 66 (2): 114-119. https://dx.doi.org/10.35366/100480

\section{RESUMEN}

Las fracturas de pelvis se asocian con una elevada mortalidad por la propia lesión y/o lesiones asociadas como cráneo, tórax, abdomen y extremidades. En particular, las lesiones inestables tipo $\mathrm{C}$ en la clasificación de Tile $\mathrm{AO}$ del anillo pélvico pueden ocasionar hemorragia masiva que en más de $80 \%$ de los casos tiene como origen el plexo presacro. Hasta $25 \%$ de estos pacientes mueren como consecuencia directa del choque hemorrágico severo. El clamp en C permite una reducción rápida, compresión y estabilización de la lesión, por tanto, es una herramienta muy útil en el control del choque, sin retrasar ni obstaculizar el diagnóstico y tratamiento definitivo, permitiendo realizar estudios como tomografía y resonancia magnética sin la necesidad de retirar el sistema, así como proporcionar acceso sin restricciones al abdomen, la pelvis o fémur proximal, sitios de lesiones concomitantes frecuentes a lesiones de pelvis.

Palabras clave: Clamp en C, fijador externo, fractura pélvica.

Nivel de evidencia: III

\begin{abstract}
Pelvic fractures are associated with high mortality due to the injury itself and/or associated injuries like head, chest, abdomen, and extremities. Unstable pelvic ring injuries can cause massive hemorrhage, more than $80 \%$ originating from the presacral plexus. Up to $25 \%$ of these patients die as a direct consequence of severe hemorrhagic shock. The C-clamp allows a rapid and efficient reduction, compression and stabilization of these injuries, therefore, it is an useful tool in shock control, without delaying or hindering the subsequent diagnosis and definite treatment; through mechanical stabilization, bleeding is controlled, thus gaining enough time for definitive control, being able to perform studies such as tomography and magnetic resonance without the need to remove the system, as well as providing unrestricted access to the abdomen, pelvis or proximal femur, injury sites frequent concomitant to pelvic injuries.
\end{abstract}

Keywords: C-clamp, external fixator, pelvis fracture.

Level of evidence: III

\section{* Cirujano Ortopedista, Centro de Ortopedia y Traumatología Centro Médico ABC. Ciudad de México, México.}

\section{Correspondencia:}

Luis Justino Fernández Palomo

E-mail: ljfernandez@abchospital.com

Recibido: 02/02/2021. Aceptado: 02/06/2021.

Abreviatura:

MAST = Military anti shock trouser

\section{INTRODUCCIÓN}

Las fracturas pélvicas inestables se asocian con una alta mortalidad por la hemorragia masiva que pueden ocasionar, siendo ésta con origen en el plexo presacro la mayoría de las veces, ${ }^{1-5}$ y aunque comprenden alrededor de $2 \%$ de todas las fracturas, su frecuencia está aumentando debido al incremento en el número de pacientes con lesiones de alta energía 
como accidentes vehiculares de alta velocidad y caídas de altura incluyendo intentos de suicidio. ${ }^{4-6}$ Los parámetros que predicen la mortalidad son la edad, la puntuación, el índice de severidad de lesión y la existencia de hemorragia grave, así como la presencia de lesiones asociadas. Las fracturas pélvicas asociadas con lesiones intracraneales o lesiones abdominales, así como las fracturas expuestas tienen tasas de mortalidad de hasta $50 \%$. $^{2,4,6,7}$

El reconocimiento inmediato del choque hemorrágico y el control eficaz del sangrado deben ser fundamentales en todo esfuerzo de reanimación. Aparte de la reposición agresiva de volumen, una parte importante del algoritmo de reanimación es la reducción y estabilización temprana de la lesión pélvica. ${ }^{7,8} \mathrm{El}$ manejo de estas lesiones potencialmente letales requiere una estabilización rápida por parte de un equipo médico de primer contacto, de personal capacitado con un protocolo de tratamiento definido principalmente por el cirujano ortopedista, cirujano general y los médicos urgenciólogos. Esto reduce el volumen intrapélvico, proporcionando un taponamiento más temprano y disminuye la pérdida de sangre al prevenir un mayor movimiento de la hemipelvis inestable y la alteración de los coágulos de sangre que ya se han formado. ${ }^{1,2,4,6,8,9}$ Esto es útil para optimizar el tiempo en el diagnóstico y tratamiento de emergencia adicionales.

Se han recomendado varias técnicas para el tratamiento de la hemorragia pélvica como el pantalón médico antichoque (MAST, por sus siglas en inglés: military anti-shock trouser), actualmente en desuso en centros de urgencia y útil en situaciones bélicas, el cual proporciona una redistribución inicial de la sangre de las extremidades al tronco y restringe la expansión del hematoma pélvico; puede ser útil para la estabilización inmediata del sangrado y para permitir el transporte del paciente del lugar del accidente a un centro de trauma. ${ }^{7,10-12}$ No es adecuado para una aplicación a largo plazo debido al riesgo de síndrome compartimental y también impide el acceso a las extremidades y al abdomen para procedimientos diagnósticos y terapéuticos adicionales, lo que dificulta la evaluación del paciente en la sala de emergencias. ${ }^{10,11}$ La fijación externa puede estabilizar la pelvis en la parte anterior y cuando se realiza temprano, disminuye el sangrado. Sin embargo, para las inestabilidades posteriores, el efecto de cualquier marco anterior es limitado y su aplicación dificulta el acceso al abdomen y la pelvis. . $^{111,13-15}$ La angiografía con embolización selectiva sólo es aplicable para hemorragias arteriales y requiere personal experimen- tado si se quieren evitar demoras en la reanimación y el tratamiento. ${ }^{16,17}$ Un método muy práctico, accesible y económico es el uso de la sábana pélvica, así como los binders o sujetadores pélvicos ajustables. Con éstos es factible disminuir la expansión de un hematoma intrapélvico al restringir el volumen de la cavidad pélvica. La pinza pélvica o clamp en $\mathrm{C}$, desarrollada por el Dr. Reinhold Ganz, es un dispositivo para abordar estos problemas. ${ }^{1,2,15,18}$

El clamp en $\mathrm{C}$ se introdujo como un dispositivo de estabilización para reducir y comprimir la pelvis posterior en pacientes con hemorragia severa. ${ }^{1,2,11,13-16,18}$ Tiene aplicaciones en la reanimación de pacientes con fracturas pélvicas inestables tipo $\mathrm{C}$, con lesión del anillo pélvico posterior como la luxación sacroiliaca o fracturas sacras verticales, donde la fijación anterior externa puede no sólo no estabilizar adecuadamente la pelvis posterior, sino incrementar el desplazamiento anterior de estas estructuras. Las indicaciones para el uso del clamp en $\mathrm{C}$ son: estabilización de emergencia de fracturas y lesiones sacroiliacas y lesiones pélvicas tipo $\mathrm{C}$ con inestabilidad hemodinámica asociada. Las contraindicaciones relativas son lesiones tipo B con estabilidad parcial lograda con fijación anterior o fracturas de sacro conminutas con riesgo de compresión del plexo del nervio sacro y las contraindicaciones absolutas son: fractura del hueso iliaco o fractura luxación transilvana (inestabilidades transiliacas) y en lesiones tipo A que por definición son fracturas estables.

La estabilización de una fractura sacra vertical con inestabilidad pélvica puede ser difícil debido al movimiento persistente en las superficies de la fractura que, en teoría, también propician otras condiciones como émbolos grasos, alteraciones respiratorias y coagulopatías. ${ }^{19,20}$ Por lo tanto, la obtención de

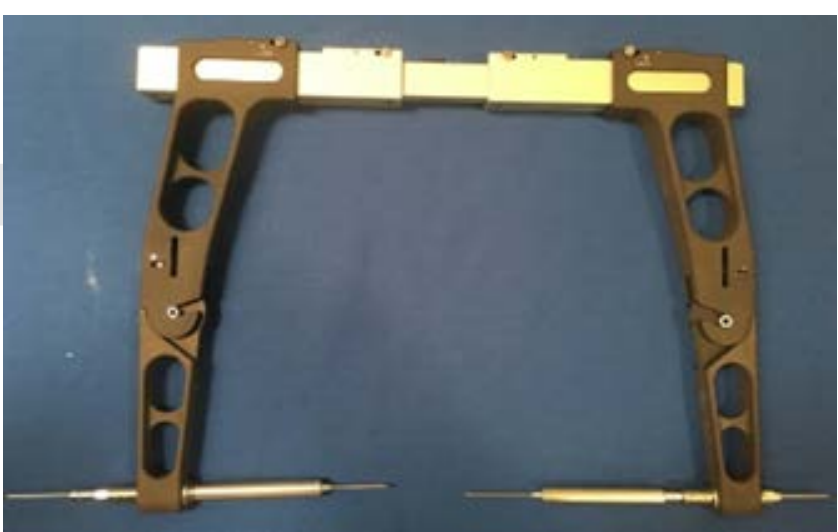

Figura 1: Clamp en C DePuy Synthes R. 


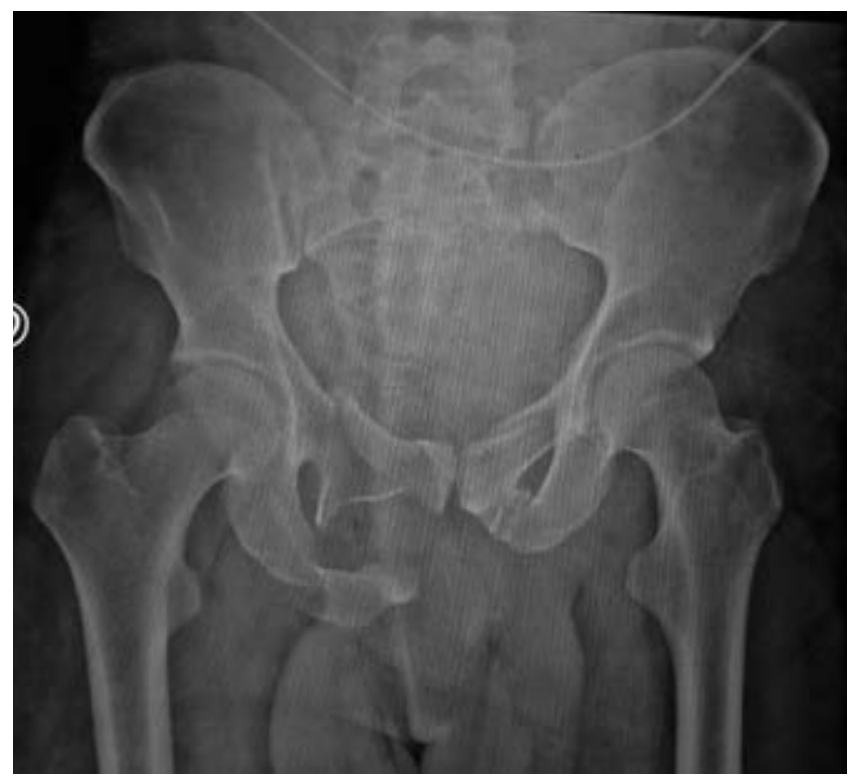

Figura 2: Radiografía anteroposterior fractura tipo C de pelvis, desplazamiento vertical fractura de sacro izquierda y de cuatro ramas anteriores.

una estabilización esquelética temporal rígida con un clamp en $\mathrm{C}$ puede mejorar las condiciones generales del paciente. Ertel y colaboradores informaron sobre 20 pacientes con fracturas pélvicas inestables en choque hemorrágico. ${ }^{13}$ Todos los pacientes se sometieron a una estabilización inmediata con un clamp en C. La tasa de mortalidad fue de $25 \%$; cuatro pacientes murieron por exanguinación en 9 horas. Sadri y su equipo revisaron 14 pacientes con fracturas inestables del anillo pélvico y choque hemorrágico tratados con estabilización inmediata con clamp en $\mathrm{C}$ más embolización, la tasa de mortalidad fue de $14 \% .{ }^{16}$

El clamp en $\mathrm{C}$ permite una estabilización esquelética rápida y control de daños, disminuyendo el riesgo de un «segundo golpe». Desde la década de los 90 se han diseñado varios modelos por diferentes casas comerciales. El primer diseño fue fabricado por Synthes de acuerdo con el prototipo de Ganz y fue conocido como el marco de Ganz o marco en C. Más tarde se introdujeron otros sistemas como el clamp en $\mathrm{C}$ de DePuy, actualmente en desuso. Hoy en día se cuenta con la nueva versión del clamp en C de DePuy Synthes, el cual es una evolución del clamp original fabricado como un dispositivo modular, ajustable más versátil y ajustable para pacientes de diferente complexión física (Figura 1). La técnica es muy similar para todos los dispositivos, siendo mucho más sencilla para su aplicación con el nuevo sistema de Synthes, el cual consta de dos puntales canulados la- terales que se pueden deslizar a lo largo de un riel con la finalidad de comprimir transversalmente la articulación sacroiliaca. En el extremo libre de cada brazo lateral hay una abertura a través de la cual se pasa un compresor roscado con un clavo interior. Un clavo de cada lado estabiliza al hueso a través de una incisión al deslizar los puntales juntos en el ala iliaca. En este punto se aplica tracción a la pierna y, si es necesario, se puede colocar un clavo de Steinmann en la cresta iliaca para manipulación adicional. A continuación, se ajusta el tubo roscado y se comprime y fija firmemente el anillo pélvico posterior. Los datos experimentales muestran que se aplica una fuerza de compresión promedio de $342 \mathrm{~N}$ al área de la junta articulación sacroiliaca. ${ }^{21,22}$

Las complicaciones de la colocación del clamp en $\mathrm{C}$ incluyen lesión de la arteria glútea superior y del nervio ciático si las puntas se insertan demasiado distales o, de colocarse de manera anterior, puede causar lesión de órganos intrapélvicos. ${ }^{23,24} \mathrm{La}$ rotación externa de la hemipelvis debe corregirse manualmente antes de colocarla, de lo contrario, aumenta el riesgo de malposición de la espiga y luxación hemipelviana, por lo que su colocación debe ser realizada por cirujanos ortopedistas traumatólogos experimentados..$^{23,25-27}$

\section{DESCRIPCIÓN DE LA TÉCNICA}

El set del clamp en $\mathrm{C}$ debe estar disponible para su uso en situaciones de emergencia extrema en el área de quirófano o urgencias.

Las referencias anatómicas como cresta iliaca o región trocantérica pueden encontrarse alteradas por la severidad de las lesiones y ser difíciles de identificar. Se recomienda el uso de intensificador

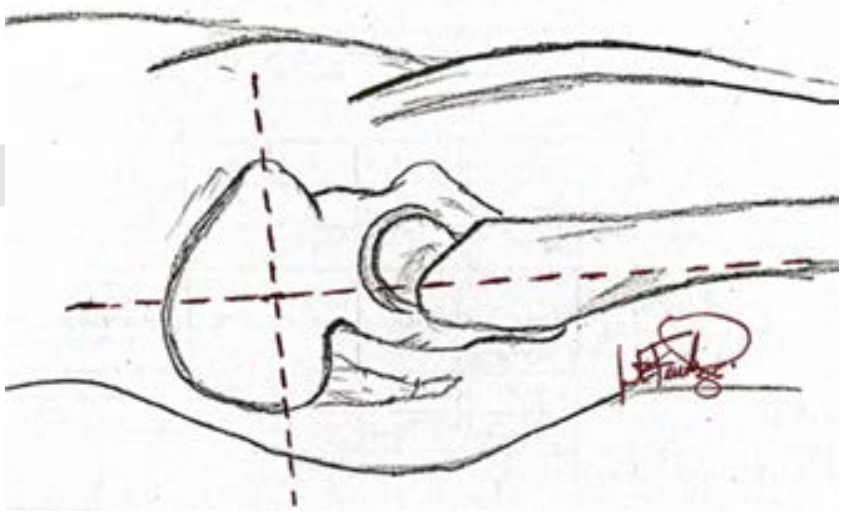

Figura 3: Identificación del sitio de colocación. 


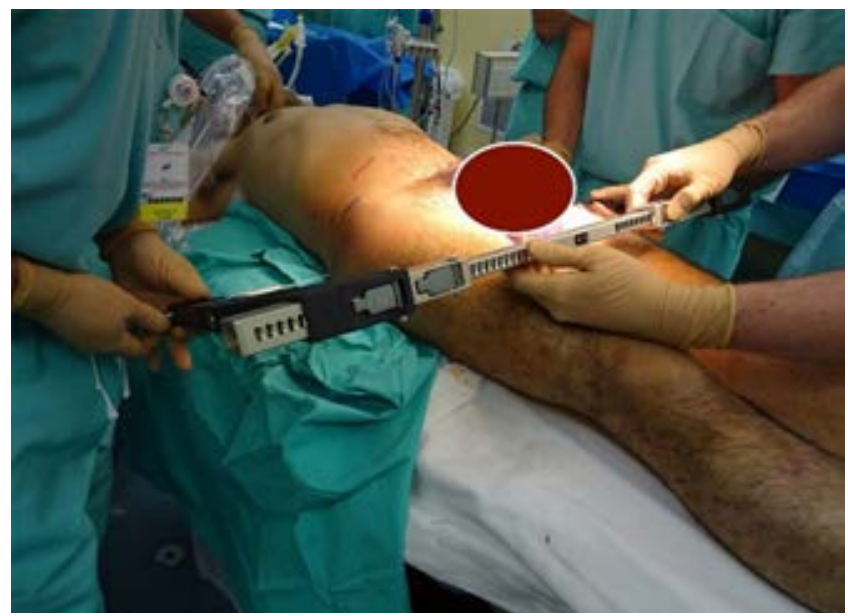

Figura 4: Colocación del sistema.

de imágenes durante la colocación del dispositivo en proyecciones anteroposterior, entrada y salida de pelvis y proyecciones oblicua alar y obturatriz (Figura 2).

El paciente se coloca en posición supina. Para facilitar la reducción se prepara el área pélvica, suprapúbica y proximal de ambos muslos. Las extremidades pélvicas deben quedar libres para facilitar su manipulación mediante tracción y rotación.

Es importante que el dispositivo sea armado en forma correcta por el personal de enfermería asistente o por el mismo equipo quirúrgico para evitar tiempo perdido. La apertura máxima del clamp facilita su colocación.

El sitio de inserción se identifica en el punto de intersección de una línea recta que sigue la dirección de la diáfisis femoral hacia la cresta iliaca y una línea perpendicular orientada de la espina iliaca anterosuperior a la espina iliaca posterosuperior (Figura 3). Se realiza una incisión de $1 \mathrm{~cm}$ y se introduce el punzón palpando la superficie lateral de la tabla externa del iliaco palpando la zona de depresión de la fosa de la tabla externa a nivel de la articulación sacroiliaca. A través del punzón se introduce un clavo de Kirchner que servirá de guía para los clavos canulados del clamp. La dirección de los clavos $\mathrm{K}$ es fundamental para evitar lesiones a estructuras anteriores y posteriores del anillo pélvico. Debe colocarse el clavo Kirchner sólo del lado afectado. La fractura debe ser reducida antes de la colocación definitiva del clamp. Con la adecuada reducción de la fractura realizada se posiciona el clavo canulado del sistema en el clavo $\mathrm{K}$ y se introduce hasta chocar con la tabla externa del iliaco. Se coloca el clavo contralateral siguiendo la misma orientación del clavo del lado de la fractura, una vez corroborada la posición de ambos y se realiza compresión del riel del clamp en C (Figura 4). Se verifica la reducción y se ajusta la posición de los clavos canulados o puntales y se corta el clavo $\mathrm{K}$ en el borde externo de este último.

Se bloquean los botones superiores del riel del clamp para evitar aflojamiento o compresión al movilizar al paciente. Se cubren las zonas de iniciación y si es necesario se coloca un punto de sutura en la misma. Se cubren con gasas estériles y se continúa el manejo de acuerdo con el protocolo de trauma para cada caso.

Se colocan compresas o un campo en la región anterior de los muslos y del abdomen para evitar el choque directo del clamp y evitar lesiones por presión (Figura 5). No debe movilizarse o levantar al paciente del clamp, situación que es común por la falta de conocimiento del personal paramédico a cargo del paciente. Se realiza una radiografía de control en proyección anteroposterior de la pelvis para verificar la posición de los clavos y la reducción de la lesión (Figura 6).

El tratamiento de pacientes hemodinámicamente inestables con lesiones del anillo pélvico es controversial. Los pacientes por lo general se encuentran en condición grave y el resultado está determinado por la complejidad y la gravedad del trauma y no debe ser atribuido a una lesión específica, ya que puede propiciarse un diagnóstico equivocado y llevar a decisiones terapéuticas erróneas. Los principales pasos del manejo primario ya están establecidos: control respiratorio, analgesia adecuada y reposición de volumen. El momento de los procedimientos invasivos como el lavado peritoneal diagnóstico, la fijación

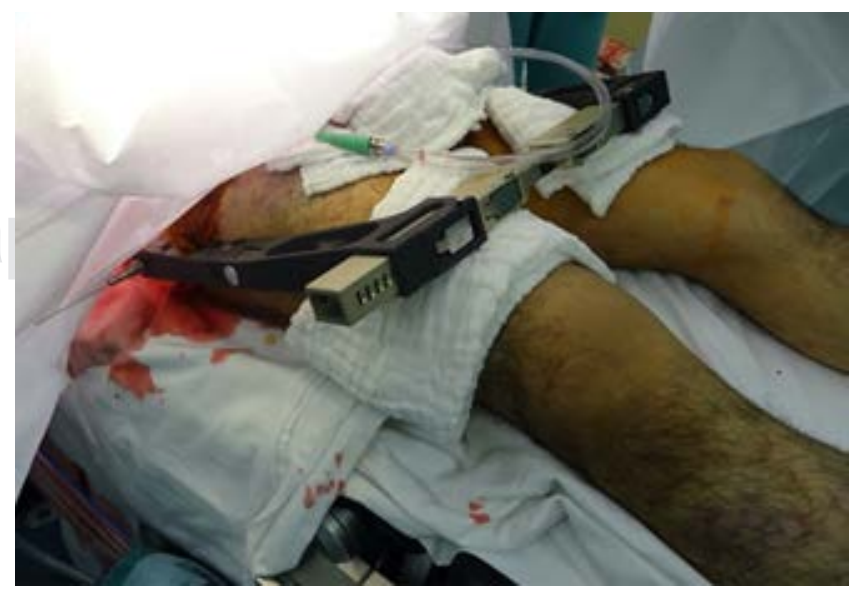

Figura 5: Postoperatorio inmediato. Protección de zonas de apoyo. 


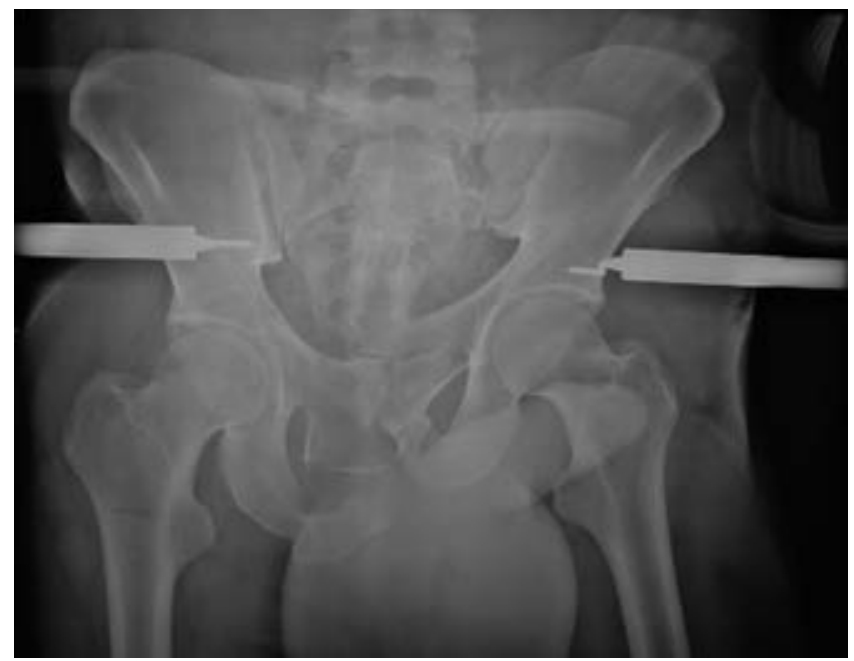

Figura 6: Radiografía anteroposterior postoperatoria. Control de desplazamiento vertical y horizontal.

externa, la angiografía y embolización pélvica son controvertidos. ${ }^{12,28,29}$

La estabilización pélvica inmediata está respaldada en la mayoría de los informes, aunque ha sido cuestionada en artículos recientes. ${ }^{3-5,12,16,18,28,29}$ Dado que la mayoría de los autores aceptan la fijación esquelética externa temprana como indicación, la discusión se centra en si es mejor un marco anterior o una pinza posterior como se describe. En lesiones del anillo posterior o con inestabilidad vertical es preferible un dispositivo posterior. ${ }^{1,2,15,16,22,26}$ También es más compleja su colocación, se requiere de entrenamiento especial y el conocimiento del dispositivo. El clamp en C combina la eficiencia mecánica con la facilidad y seguridad de uso, permitiendo un fácil acceso a la región abdominal para un diagnóstico y tratamiento adicionales. ${ }^{30-32}$

\section{CONCLUSIÓN}

Las lesiones y fracturas inestables del anillo pélvico se asocian con hemorragia masiva que puede causar choque hemorrágico severo. El clamp pélvico en $\mathrm{C}$ es un fijador externo de estabilización de emergencia para lesiones inestables y fracturas del anillo pélvico con compromiso de estructuras posteriores que permite una rápida y eficiente reducción, compresión y estabilización de las mismas. Es útil para obtener el control del choque, sin retrasar ni obstaculizar el diagnóstico y tratamientos concomitantes; mediante la estabilización mecánica se controla la hemorragia, ganando así tiempo suficiente para el control defini- tivo, permitiendo realizar estudios como tomografía axial, angiografía o ultrasonido sin la necesidad de retirar el sistema, así como proporcionar acceso sin restricciones al abdomen, la pelvis o fémur proximal.

\section{REFERENCIAS}

1. Ganz R, Krushell RJ, Jakob RP, Kuffer J. The antishock pelvic clamp. Clin Orthop Relat Res. 1991; (267): 71-78.

2. Gansslen A, Lindahl J. The history of pelvic fracture treatment. In: Gansslen A, Lindahl J, Grechenig S, Fuchtmeier B, eds. Pelvic ring fractures. Cham, Germany: Springer International Publishing; 2021. pp. 3-14.

3. Balogh Z, King KL, Mackay P, McDougall D, Mackenzie S, Evans JA et al. The epidemiology of pelvic ring fractures: a population-based study. J Trauma. 2007; 63 (5): 1066-1073; discussion 1072-1073.

4. Pohlemann T, Tscherne H, Baumgartel F, Egbers HJ, Euler $\mathrm{E}$, Maurer F et al. Pelvic fractures: epidemiology, therapy and long-term outcome. Overview of the multicenter study of the Pelvis Study Group. Unfallchirurg. 1996; 99 (3): 160-167.

5. Giannoudis PV, Grotz MR, Tzioupis C, Dinopoulos H, Wells GE, Bouamra O et al. Prevalence of pelvic fractures, associated injuries, and mortality: the United Kingdom perspective. J Trauma. 2007; 63 (4): 875-883.

6. Tosounidis G, Holstein JH, Culemann U, Holmenschlager F, Stuby F, Pohlemann T. Changes in epidemiology and treatment of pelvic ring fractures in Germany: an analysis on data of German Pelvic Multicenter Study Groups I and III (DGU/AO). Acta Chir Orthop Traumatol Cech. 2010; 77 (6): 450-456.

7. Holstein JH, Culemann U, Pohlemann T; Working Group Mortality in Pelvic Fracture Patients. What are predictors of mortality in patients with pelvic fractures? Clin Orthop Relat Res. 2012; 470 (8): 2090-2097.

8. Rommens PM, Hofmann A, Hessmann MH. Management of acute hemorrhage in pelvic trauma: an overview. Eur J Trauma Emerg Surg. 2010; 36 (2): 91-99.

9. Vallier HA, Cureton BA, Ekstein C, Oldenburg FP, Wilber JH. Early definitive stabilization of unstable pelvis and acetabulum fractures reduces morbidity. J Trauma. 2010; 69 (3): 677-684.

10. Routt ML Jr, Falicov A, Woodhouse E, Schildhauer TA. Circumferential pelvic antishock sheeting: a temporary resuscitation aid. J Orthop Trauma. 2006; 20 (1 Suppl): S3-S6.

11. Warme WJ, Todd MS. The circumferential antishock sheet. Mil Med. 2002; 167 (5): 438-441.

12. Kortbeek JB, Al Turki SA, Ali J, Antoine JA, Bouillon B, Brasel $\mathrm{K}$ et al. Advanced trauma life support, 8th edition, the evidence for change. J Trauma. 2008; 64 (6): 1638-1650.

13. Ertel W, Keel M, Eid K, Platz A, Trentz O. Control of severe hemorrhage using C-clamp and pelvic packing in multiply injured patients with pelvic ring disruption. J Orthop Trauma. 2001; 15 (7): 468-474.

14. Jowett AJ, Bowyer GW. Pressure characteristics of pelvic binders. Injury. 2007; 38 (1): 118-121.

15. Tiemann AH, Bohme J, Josten C. Use of the pelvic clamp in polytraumatised patients with unstable disruption of the posterior pelvic ring. Modified technique--risks--problems. Orthopade. 2006; 35 (12): 1225-1236.

16. Sadri H, Nguyen-Tang T, Stern R, Hoffmeyer P, Peter R. Control of severe hemorrhage using C-clamp and arterial embolization in hemodynamically unstable patients with pelvic ring disruption. Arch Orthop Trauma Surg. 2005; 125 (7): 443-447. 
17. Culemann U, Tosounidis G, Reilmann H, Pohlemann T. Injury to the pelvic ring. Diagnosis and current possibilities for treatment. Unfallchirurg. 2004; 107 (12): 1169-1181; quiz 1182-1183.

18. Heini PF, Witt J, Ganz R. The pelvic C-clamp for the emergency treatment of unstable pelvic ring injuries. A report on clinical experience of 30 cases. Injury. 1996; 27 Suppl 1: 38-45.

19. Quintero AJ, Tarkin IS, Pape HC. Case report: The prone reduction of a sacroiliac disruption with a pelvic C-clamp. Clin Orthop Relat Res. 2009; 467 (4): 1103-1106.

20. Wiznia DH, Swami N, Kim CY, Leslie MP. A method of using a pelvic c-clamp for intraoperative reduction of a zone 3 sacral fracture. Case Rep Orthop. 2016; 2016: 8586517.

21. Lustenberger T, Meier C, Benninger E, Lenzlinger PM, Keel MJ. C-clamp and pelvic packing for control of hemorrhage in patients with pelvic ring disruption. J Emerg Trauma Shock. 2011; 4 (4): 477-482.

22. Krieg JC, Mohr M, Ellis TJ, Simpson TS, Madey SM, Bottlang M. Emergent stabilization of pelvic ring injuries by controlled circumferential compression: a clinical trial. J Trauma. 2005; 59 (3): 659-664.

23. Koller H, Balogh ZJ. Single training session for first time pelvic C-clamp users: correct pin placement and frame assembly. Injury. 2012; 43 (4): 436-439.

24. Bartlett C, Asprinio D, Louis S, Helfet D. Intrapelvic dislocation of the left hemipelvis as a complication of the pelvic "C" clamp: a case report and review. J Orthop Trauma. 1997; 11 (7): 540-542.

25. Pohlemann T, Braune C, Gansslen A, Hüfner T, Partenheimer A. Pelvic emergency clamps: anatomic landmarks for a safe primary application. J Orthop Trauma. 2004; 18 (2): 102-105.

26. Reynolds JH, Attum B, Acland RJ, Giannoudis P, Roberts CS. Anterior versus posterior pin placement of pelvic C-clamp in relationship to anatomical structures: a cadaver study. Injury. 2008; 39 (8): 865-868.

27. Spanjersberg WR, Knops SP, Schep NW, van Lieshout EM, Patka P, Schipper IB. Effectiveness and complications of pelvic circumferential compression devices in patients with unstable pelvic fractures: a systematic review of literature. Injury. 2009; 40 (10): 1031-1035.

28. Giannoudis PV. Surgical priorities in damage control in polytrauma. J Bone Joint Surg Br. 2003; 85 (4): 478-483.

29. Giannoudis PV, Pape HC. Damage control orthopaedics in unstable pelvic ring injuries. Injury. 2004; 35 (7): 671-677.

30. Fernández-Palomo LJ. Fracturas de pelvis en pacientes hemodinámicamente inestables. Acta Ortop Mex. 2012; 26 (5): 275-281.

31. Fernández PLJ, Fernández VJM. Evaluación radiográfica de la pelvis. An Med Asoc Med Hosp ABC. 2002; 47 (2): 91-97.

32. Sommer G, Fernández LJ. Evaluación y manejo inicial de las fracturas inestables de pelvis. En: Serrano Ardilla AM, Camacho Galindo J. El ABC de la traumatología. Temas selectos. Ed. Alfil; 2020. pp. 109-118. 